\title{
Distribution of branchial anomalies in a paediatric Asian population
}

\author{
Neville Wei Yang ${\underline{T e o^{1}}}^{1}$ MBBS, MRCS, Shahrul Izham $\underline{\mathrm{Ibrahim}^{2}}$, MBBCh, MRCs, Kun Kiaang Henry $\underline{\operatorname{Tan}^{2}}$, MD, FRCS
}

\begin{abstract}
INTRODUCTION The objective of the present study was to review the distribution and incidence of branchial anomalies in an Asian paediatric population and highlight the challenges involved in the diagnosis of branchial anomalies. METHODS This was a retrospective chart review of all paediatric patients who underwent surgery for branchial anomalies in a tertiary paediatric hospital from August 2007 to November 2012. The clinical notes were correlated with preoperative radiological investigations, intraoperative findings and histology results. Branchial anomalies were classified based on the results of the review.

RESULTS A total of 28 children underwent surgery for 30 branchial anomalies during the review period. Two children had bilateral branchial anomalies requiring excision. Of the 30 branchial anomalies, 7 (23.3\%) were first branchial anomalies, 5 (16.7\%) were second branchial anomalies, 3 (10.0\%) were third branchial anomalies, and 4 (13.3\%) were fourth branchial anomalies (one of the four patients with fourth branchial anomalies had bilateral branchial anomalies). In addition, seven children had $8(26.7 \%)$ branchial anomalies that were thought to originate from the pyriform sinus; however, we were unable to determine if these anomalies were from the third or fourth branchial arches. There was inadequate information on the remaining $3(10.0 \%)$ branchial anomalies for classification.

CONCLUSION The incidence of second branchial anomalies appears to be lower in our Asian paediatric population, while that of third and fourth branchial anomalies was higher. Knowledge of embryology and the related anatomy of the branchial apparatus is crucial in the identification of the type of branchial anomaly.
\end{abstract}

Keywords: branchial cleft, branchial cyst, branchial sinus, paediatric

\section{INTRODUCTION}

Branchial apparatus anomalies are the second most common head and neck congenital lesions seen in children, accounting for $20 \%$ of cervical masses seen in children. ${ }^{(1)}$ Second branchial cleft anomalies have been reported to be the most common branchial anomaly, accounting for up to $95 \%$ of these lesions..$^{(2,3)}$ In contrast, first branchial cleft anomalies make up only $1 \%$ of these lesions, while third and fourth branchial arch anomalies account for $3 \%-10 \%$ of these lesions. ${ }^{(4-7)}$ These anomalies may occur as cysts, sinuses or fistulae. Of these, cysts tend to present later, as they may take a longer time to enlarge and present clinically. ${ }^{(4)}$ Occasionally, the anomalies may present acutely due to superimposed infection. Despite the prevalence of such anomalies, diagnosis is not always straightforward, as clinicians may not consider them in the differential diagnosis. Even if they do, the characterisation of an anomaly to a particular branchial cleft may be difficult, or may not even be attempted for various reasons.

The embryo in utero has six branchial arches that appear by the fourth and fifth week of development; these branchial arches are separated by four ectodermal-lined branchial clefts and five endodermal-lined branchial pouches. Each branchial arch contains mesenchymal and neural crest cells, which contribute to the skeletal components of the face. Each arch contains its own dominant artery, nerve, musculature and cartilage. Similarly, each of the branchial pouches develops into a specific organ of the human body. Of the four branchial clefts, only the first one persists to contribute to the external auditory meatus and tympanic membrane. The second, third and fourth clefts are slowly obliterated as the mesenchymal tissue of the second arch proliferates and overgrows them. They form an ectodermallined cavity called the cervical sinus of His, which subsequently disappears with further development.

While the underlying pathogenesis of branchial anomalies is not entirely certain, there are theories that attempt to explain their development. These include the branchial apparatus theory, cervical sinus theory, thymopharyngeal duct theory and inclusion theory. ${ }^{(8)}$ The most widely accepted theory is that branchial anomalies are remnants from incomplete obliteration of the branchial apparatus. The resulting malformation may present as a first, second, third or fourth branchial anomaly. Differentiation of these anomalies requires intimate knowledge of the embryological development and anatomy of the head and neck. This can sometimes be difficult, even with the plethora of investigations available. The present study sought to review the distribution of branchial anomalies in an Asian paediatric population and highlight the challenges involved in the diagnosis of branchial anomalies.

\section{METHODS}

This was a retrospective chart review of paediatric patients who underwent surgery for branchial anomalies from August 2007 to November 2012 in KK Women's and Children's Hospital $(\mathrm{KKH})$, Singapore, a tertiary paediatric hospital. All paediatric

${ }^{1}$ Department of Otolaryngology, Singapore General Hospital, ${ }^{2}$ Department of Otolaryngology, KK Women's and Children's Hospital, Singapore

Correspondence: Dr Neville Teo Wei Yang, Associate Consultant, Department of Otolaryngology, Singapore General Hospital, Academia, Level 5, 20 College Road, Singapore 169856. neville.teo.w.y@sgh.com.sg 
patients treated for branchial anomalies during the study period were included.

The list of patients was retrieved from the hospital's electronic medical records. The patients' presenting history, physical findings and scans from preoperative imaging were examined. The operative records of the patients were studied in order to retrieve the intraoperative findings, which were crucial in determining the level of branchial anomalies. Histology results were also reviewed to ensure that they corresponded to the clinical findings in each case. The collected data was independently analysed by two of the authors involved in the study. Classification of the level of branchial anomaly was performed based on the data collected, with the greatest reliance placed on the operative findings and postoperative surgical diagnosis provided by the operating surgeon. In cases where seemingly conflicting results among the clinical, radiological and operative findings were found, the operative records, if sufficiently detailed, were used as the final diagnosis. Approval for the present study was obtained from the local Institutional Review Board.

First branchial cleft anomalies were easily distinguishable from other anomalies, as they typically occur around the ear. In comparison, second, third and fourth branchial anomalies tend to occur lower in the neck. While these branchial anomalies are classically distinguished by their course as well as internal and external openings, both the internal opening in the pharynx and the external opening in the neck may not always be present. In cases where the collated data was inconclusive, they were reflected as such.

\section{RESULTS}

A total of 28 children (14 male, 14 female) underwent surgery for 30 branchial anomalies during the review period, which lasted for five years and four months. Two children had excision of bilateral branchial anomalies. The age of the children at the time of surgery was 1-18 years. All 28 children were of Asian ethnicity 15 Chinese, 6 Malay, 2 Indian, 2 Sikh, 2 Indonesian and 1 Cambodian). The procedures were performed by either a paediatric surgeon or paediatric otolaryngologist at $\mathrm{KKH}$.

Of the 30 branchial anomalies, 7 (23.3\%) were first branchial anomalies, $5(16.7 \%)$ were second branchial anomalies, $3(10.0 \%)$ were third branchial anomalies, and 4 (13.3\%) were fourth branchial anomalies (one of the four patients with fourth branchial anomalies had bilateral branchial anomalies). Seven patients had $8(26.7 \%)$ branchial anomalies that were thought to originate from the pyriform sinus; however, we were unable to determine if these anomalies were from the third or fourth branchial arches. There was inadequate information on the remaining 3 (10.0\%) branchial anomalies for classification.

Of the seven patients who had eight branchial anomalies originating from the pyriform sinus, all but one had internal openings in the pyriform sinus. The single branchial sinus without an internal opening in the pyriform sinus was found in a child who had bilateral branchial sinuses; the fistula on the right branchial sinus opened into the pyriform sinus, while the tract on the left did not. All seven patients presented with infections of the branchial anomalies. Five patients had a cystic mass or abscess within the neck; three of these five abscesses were adjacent to the upper pole of the thyroid gland. As the exact courses of the tracts of these branchial anomalies were not identifiable intraoperatively, we were unable to make a definite diagnosis of their origins (i.e. from the third or fourth branchial arch).

All the seven patients with first branchial cleft anomalies presented with swelling at the postauricular region. None of them had an external sinus opening, and only one patient was found to have an internal opening in the external auditory canal. Half of these seven patients presented with either persistent or recurrent infections of the postauricular abscesses, which prompted the consideration of first branchial cleft cysts. 2 (28.6\%) patients did not present with infection of the first branchial cleft cyst.

Imaging assessment was obtained for 18 of the 30 branchial cleft anomalies that were surgically treated. The remaining branchial cleft anomalies underwent surgical treatment based on their clinical presentations. Paediatric otolaryngologists ordered imaging for 12 of the 15 (80.0\%) patients seen by them, while paediatric surgeons did so for 5 of their $13(38.5 \%)$ patients. The type of imaging assessment performed included computed tomography (CT), ultrasonography (US) and magnetic resonance (MR) imaging. The main imaging modality used was CT $(82.4 \%)$, followed by US (23.5\%) and MR imaging (11.8\%). In three patients, more than one imaging modality was used; two patients underwent both CT and US, while one patient underwent $\mathrm{CT}$ and MR imaging. Accurate diagnosis of the branchial cleft malformation was made via imaging in 16 of the 18 (88.9\%) cases that underwent preoperative imaging. The imaging reports of the remaining two cases noted the presence of a neck abscess, with no mention of the possibility of a branchial cleft anomaly. Imaging was accurate in predicting the correct level of branchial cleft malformation in 13 of the 18 (72.2\%) cases.

\section{DISCUSSION}

Second branchial anomalies are believed to be the most common type of branchial anomaly, accounting for up to $95 \%$ of all branchial apparatus anomalies; first branchial anomalies have been reported to make up only $1 \%$ of all branchial apparatus anomalies, while third and fourth branchial anomalies are reportedly even rarer than first branchial anomalies. ${ }^{(2-7)}$ However, on reviewing the published literature that focused specifically on paediatric patients, we found that this frequently reported distribution may not be accurately reflected in paediatric populations. ${ }^{(1,4,9,10)}$

Table I compares some of the findings of relevant studies with those of the present study. First branchial cleft anomalies were found to regularly account for over $15 \%$ of branchial anomalies in paediatric studies. ${ }^{(1,4,9,10)}$ Our findings, however, differ from those of these studies, in that second branchial anomalies occur less commonly in our paediatric patients. The distribution of third and fourth branchial anomalies in the present study appears to be similar to that of these studies. However, if the group of patients who had undetermined third or fourth branchial anomalies were included in the analysis, the results would suggest a much higher 
Table I. Comparison of the reported distributions of branchial anomalies in paediatric populations.

\begin{tabular}{|c|c|c|c|c|c|c|}
\hline \multirow[t]{2}{*}{ Study, year } & \multirow{2}{*}{$\begin{array}{l}\text { Duration } \\
\text { (yr) }\end{array}$} & \multirow{2}{*}{$\begin{array}{l}\text { No. of } \\
\text { cases }\end{array}$} & \multicolumn{4}{|c|}{ Branchial anomaly (\% of cases) } \\
\hline & & & First & Second & Third & Fourth \\
\hline Choi and Zalzal, 1995(4) & 10 & $52^{*}$ & 25.0 & 40.4 & 7.7 & 1.9 \\
\hline Nicollas et al, $2000^{(9)}$ & 15 & 64 & 31.3 & 57.8 & 0 & 10.9 \\
\hline Schroeder et al, $2007^{(10)}$ & 10 & 69 & 18.8 & 73.9 & 7.2 & 0 \\
\hline Bajaj et al, 2011(1) & 10 & 80 & 18.8 & 77.5 & 0 & 3.8 \\
\hline
\end{tabular}

*13 $(25.0 \%)$ cases were of undetermined origin. $\$ 3(10.0 \%)$ cases were of undetermined origin; $8(26.7 \%)$ cases had internal openings in the pyriform sinus, but whether they were from the third or fourth branchial apparatus could not be determined.

incidence of these branchial anomalies in our local paediatric population.

Work classified first branchial cleft anomalies into type I and type II. ${ }^{(11)}$ The former is defined to be of ectodermal origin, typically extending to the postauricular crease, and is considered a duplication of the membranous external auditory canal (Fig. 1). The latter is defined to be of ectodermal and mesodermal origin, and may present as an abscess below the angle of the mandible; it is considered a duplication anomaly of the membranous external auditory canal and pinna. First branchial cleft anomalies are thought to be relatively uncommon, reportedly accounting for only $1 \%-4 \%$ of all branchial anomalies. ${ }^{(1,5)}$ These should be differentiated from preauricular sinuses, as their embryology and treatment are distinct. Preauricular sinuses arise from abnormalities in auricular development. Two theories have been postulated to explain this, namely incomplete fusion of the hillocks of His (which arise from the first two branchial arches) and ectodermal folding. Confusing a first branchial cleft anomaly for a preauricular sinus or cyst is potentially dangerous; such a mistake could result in injury to the facial nerve, or incomplete excision.

Other studies have reported higher incidences of first branchial cleft anomalies. ${ }^{(4,9)}$ Similarly, the present study found a high incidence of first branchial anomalies (23.3\%). This higherthan-expected rate of occurrence is important, as clinicians treating cystic lesions or abscesses at the postauricular and periparotid regions should be aware of the possibility of a first branchial cleft anomaly, especially if infection is recurrent or persistent despite previous incision and drainage procedures. Common differentials for first branchial anomalies include epidermal cysts, dermoid cysts, mastoid abscesses, postauricular lymphadenopathy and parotid lesions.

Second branchial cleft anomalies were first described by Bailey in 1929. ${ }^{(12)}$ They were classified into the following four types: (a) type I is found deep to the platysma and anterior to the sternocleidomastoid; (b) type II lies anterior to the sternocleidomastoid, lateral to the carotid space and posterior to the submandibular gland (Fig. 2); (c) type III extends medially between the internal and external carotid arteries; and (d) type IV abuts the pharyngeal mucosal wall and may extend superiorly to the skull base. ${ }^{(12)}$ Traditionally, second branchial cleft anomalies are reported to be the most common type of branchial cleft malformation, with rates ranging up to $95 \% .^{(2,3)}$ In the present study, only $16.7 \%$ of the anomalies arose from the second branchial cleft, and all the second branchial cleft anomalies were

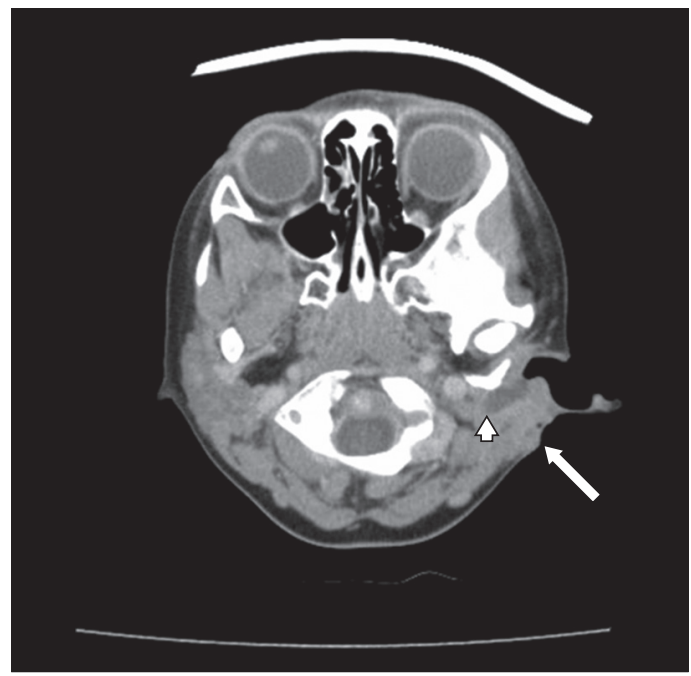

Fig. 1 Axial CT image of a first branchial cleft cyst shows external pus discharge (arrow) and a deeper abscess cavity along the external auditory meatus (arrowhead).

branchial cysts, with no sinus tract communicating intraorally. One possible explanation for the significantly lower incidence of second branchial anomalies in our paediatric study population is that branchial cysts may present later in life, as it takes a longer time for them to increase in size and to become clinically significant. We are unable to explain the absence of second branchial sinuses or fistulae in our study population; our results may have been skewed due to the relatively small number of patients in the study.

Third branchial arch anomalies originate from the base of the pyriform sinus. They pierce the inferior constrictor medial to the greater cornu of the hyoid bone and lateral to the superior laryngeal nerve, before looping over the hypoglossal nerve below the glossopharyngeal nerve. They then course inferiorly, posterior to the internal carotid artery and anterior to the vagus nerve. If present, the external opening can be found over the anterior border of the sternocleidomastoid muscle, between the middle and lower third. ${ }^{(13,14)}$ In theory, fourth branchial arch anomalies follow a more convoluted route than third branchial arch anomalies. The former originate from the apex of the pyriform sinus medial to the superior laryngeal nerve, and travel inferiorly in the tracheoesophageal groove, behind the thyroid gland and parallel to the recurrent laryngeal nerve into the mediastinum, where they loop around the aorta on the left and subclavian artery on the right. They then ascend into the neck, posterior to the common carotid artery to reach the hypoglossal nerve, where 

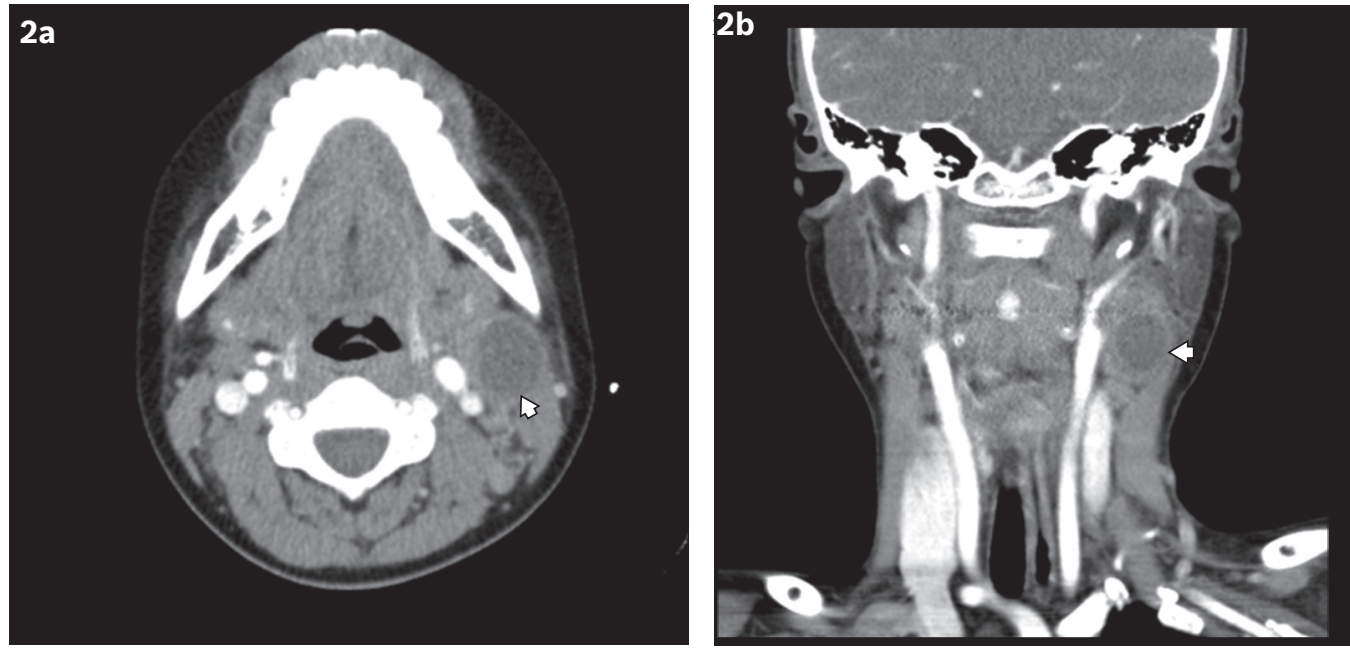

Fig. 2 (a) Axial and (b) coronal CT images show a second branchial cleft cyst (arrowheads) located medial to sternocleidomastoid muscle and lateral to the carotid sheath.
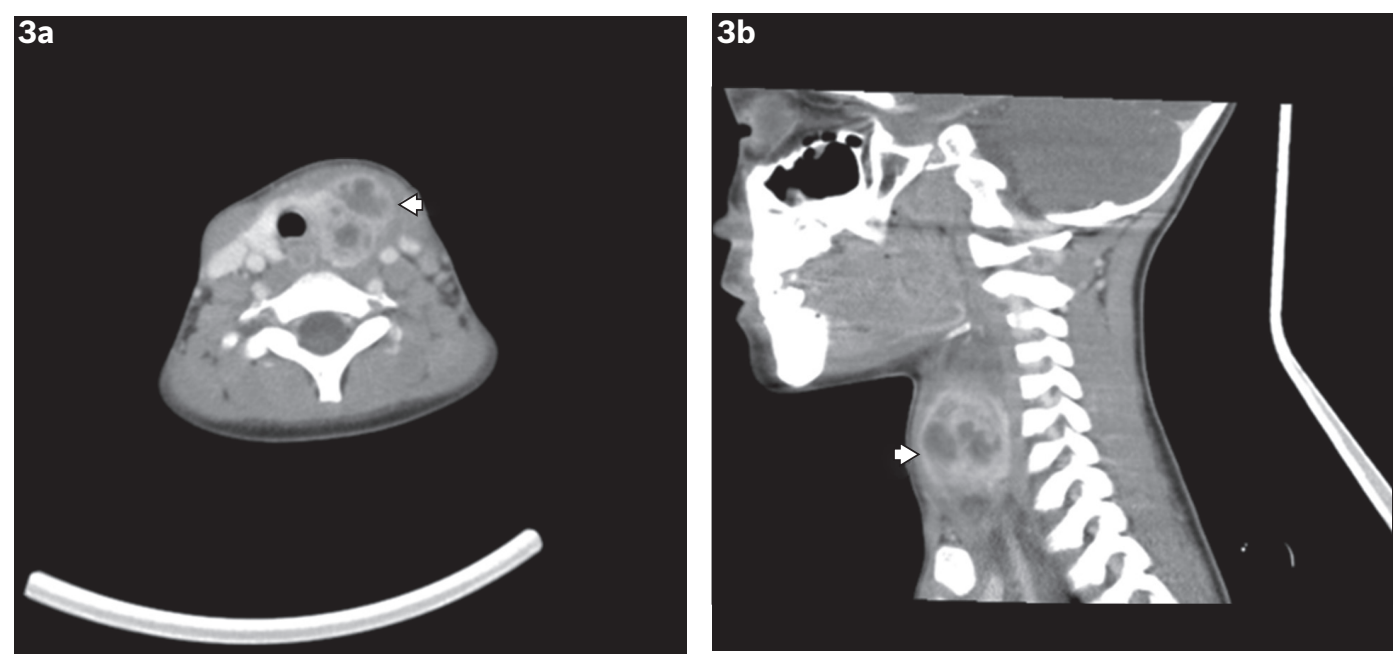

Fig. 3 (a) Axial and (b) sagittal CT images of a fourth branchial cleft cyst show the cyst involving the left thyroid lobe (arrowheads).

a second loop is made around the nerve to end at the anterior border of the sternocleidomastoid muscle. ${ }^{(6,14)}$ In view of their close proximity to the thyroid gland, third and fourth branchial anomalies can sometimes present as suppurative thyroiditis or intrathyroidal abscesses (Fig. 3).

In the present study, third and fourth branchial arch anomalies accounted for $10.0 \%$ and $13.3 \%$ of the branchial anomalies, respectively. Third and fourth branchial arch anomalies were distinguished by observing the aforementioned anatomical differences in their tracts. Together with the seven patients with branchial sinuses opening into the pyriform sinus, branchial anomalies originating from the third and fourth branchial arches were found in 13 of the $28(46.4 \%)$ patients. This incidence is significantly higher than that reported in the literature thus far. As compared to the studies listed in Table I, only Asian patients were included in the present study, suggesting that there may be a higher incidence of third and fourth branchial anomalies among Asian children. However, further studies are needed to confirm this, as our study population was not representative of all Asian nationalities and ethnicities. Although there was one published study that investigated congenital cysts in Taiwanese children, ${ }^{(15)}$ it did not provide information on the distribution of branchial anomalies.

Imaging yields a high rate of preoperative diagnosis of branchial anomalies, and this was true in the present study. CT and MR imaging are able to provide excellent anatomical detail, while US has the advantage of greater tolerability by children and no exposure to ionising radiation (thus allowing serial monitoring of the size and character of the cystic masses). Sedation may be required for younger children undergoing MR imaging, as they may be unable to cooperate with the examination requirements. We were not able to compare the accuracy of the imaging modalities due to the small number of patients who underwent US and MR imaging.

Several methods can be used to identify the presence of internal openings of branchial anomalies, if any. These include radiographic imaging (e.g. contrast swallow or fistulograms), or direct visualisation using a flexible nasolaryngoscope, an intraoperative laryngoscope or an oesophageoscope. Occasionally, probing of the tract from its external opening 
may also be helpful. Contrast swallow studies have previously been performed for the workup of branchial anomalies to detect communications with the upper aerodigestive tract. While such studies may be useful, very young children may not be cooperative.

Fistulograms have been found to be useful for differentiating sinus tracts from complete fistulae, and for delineating the length and course of the tracts. ${ }^{(16)}$ They are simple and safe, and can be performed either pre- or intraoperatively. Fistulograms are especially helpful if there is a need to excise an inflamed tract in its entirety (due to inflammation obscuring the dissection planes), and they also prevent excessive removal of normal tissue surrounding the tract. If performed preoperatively, they can aid in surgical planning. However, fistulograms may not be tolerated by all children, and they may not be necessary if the tract has been clearly identified via other forms of imaging modality or direct visualisation, or by probing with a lacrimal probe or umbilical catheter. In the present study, none of our paediatric patients underwent contrast swallow studies or fistulograms. However, intraoperative oesophagoscopy and laryngoscopy were performed, when indicated, to look for internal openings of the sinus tracts; this was done in children suspected to have third or fourth branchial anomalies.

The greatest clinical utility of preoperative imaging is in the management of first branchial cleft anomalies. This is due to the occasional innocuous presentation of first branchial cleft anomalies and their intimate association with facial nerve. CT or MR imaging can help to not only confirm the clinical suspicion of first branchial cleft anomalies, but also warn parents and patients regarding the risk of facial nerve injury and the extent of surgery required. In the present study, all but one of the six patients with first branchial anomalies underwent CT imaging preoperatively. This has helped to confirm the diagnosis and aid surgical planning. While it may not always be possible or feasible to perform preoperative imaging, especially in emergency situations or when facilities are unavailable, it should always be considered to aid diagnosis and surgical planning. Clinicians should weigh the risks and benefits before making a final decision on whether preoperative imaging should be performed, and if so, which imaging modality would be most appropriate.

The present study was not without limitations. The first being the relatively small number of patients in our study population, which may have affected the distribution of branchial anomalies, thus accounting for the lower rate of second branchial anomalies and higher rates of third and fourth branchial anomalies. We were also unable to determine the superiority of a single imaging modality in diagnosing branchial anomalies, again due to the small cohort, although this was not the primary intent of the present study. Continued data collection from the centre will help to better elucidate the true incidence of the various branchial anomalies in our local Asian population.

In conclusion, the results of the present study suggest that first, third and fourth branchial apparatus anomalies may present much more frequently in the paediatric population than commonly believed. Third and fourth branchial arch anomalies appear to occur more often in our local Asian population, although further studies are needed to ascertain this finding. Imaging has been shown to be a useful adjunct in the management of these congenital lesions.

\section{REFERENCES}

1. Bajaj $Y$, Ifeacho S, Tweedie D, et al. Branchial anomalies in children. Int J Pediatr Otorhinolaryngol 2011; 75:1020-3.

2. Kenealy JF, Torsiglieri AJ Jr, Tom LW. Branchial cleft anomalies: a fiveyear retrospective review. Trans Pa Acad Ophthalmol Otolaryngol 1990; 42:1022-5.

3. Acierno SP, Waldhausen JHT. Congenital cervical cysts, sinuses and fistulae. Otolaryngol Clin North Am 2007; 40:161-76, vii-viii.

4. Choi SS, Zalzal GH. Branchial anomalies: a review of 52 cases. Laryngoscope 1995; 105:909-13.

5. Mounsey RA, Forte V, Friedberg J. First branchial cleft sinuses: an analysis of current management strategies and treatment outcomes. J Otolaryngol 1993; 22:457-61.

6. Nicoucar K, Giger R, Pope HG Jr, Jaecklin T, Dulguerov P. Management of congenital fourth branchial arch anomalies: a review and analysis of published cases. J Pediatr Surg 2009; 44:1432-9.

7. Pereira KD, Losh GG, Oliver D, Poole MD. Management of anomalies of the third and fourth branchial pouches. Int J Pediatr Otorhinolaryngol 2004; 68:43-50

8. Chandler JR, Mitchell B. Branchial cleft cysts, sinuses, and fistulas. Otolaryngol Clin North Am 1981; 14:175-86.

9. Nicollas R, Guelfucci B, Roman S, Triglia JM. Congenital cysts and fistulas of the neck. Int J Pediatr Otorhinolaryngol 2000; 55:117-24.

10. Schroeder JW Jr, Mohyuddin N, Maddalozzo J. Branchial anomalies in the pediatric population. Otolaryngol Head Neck Surg 2007; 137:289-95.

11. Work WP. Newer concepts of first branchial cleft defects. Laryngoscope 1972; 82:1581-93.

12. Bailey H. Branchial Cysts and Other Essays on Surgical Subjects in the Facio-cervical Region. London: H.K Lewis \& Company, 1929.

13. Nicoucar K, Giger R, Jaecklin T, Pope HG Jr, Dulguerov P. Management of congenital third branchial arch anomalies: a systematic review. Otolaryngol Head Neck Surg 2010; 142:21-28.e2.

14. James A, Stewart C, Warrick P, Tzifa C, Forte V. Branchial sinus of the piriform fossa: reappraisal of third and fourth branchial anomalies. Laryngoscope 2007; 117:1920-4.

15. Hsieh YY, Hsueh S, Hsueh C, et al. Pathological analysis of congenital cervical cysts in children: 20 years of experience at Chang Gung Memorial Hospital. Chang Gung Med J 2003; 26:107-13.

16. Guarisco JL, Fatakia A. Intraoperative fistulograms in the management of branchial apparatus abnormalities in children. Int J Pediatr Otorhinolaryngol 2008; 72:1777-82. 